\title{
Bright and dark rogue internal waves: The Gardner equation approach
}

\author{
Mahyar Bokaeeyan," A. Ankiewicz, and N. Akhmediev \\ Optical Sciences Group, Research School of Physics and Engineering, The Australian National University, Canberra, ACT 2600, Australia
}

(Received 25 March 2019; published 25 June 2019)

\begin{abstract}
We have found "bright and dark" solutions of the Gardner equation which can model internal rogue waves in three-layer fluids. We provide the first four "bright" and "dark" exact rational solutions to the Gardner equation. These are the lowest-order solutions of the corresponding hierarchies of rogue-wave solutions of this equation. They have been obtained from the rogue-wave solutions of a modified Korteweg-de Vries equation by using a Lorentz-type transformation. The maximal (and minimal) amplitudes and the background levels of these solutions for arbitrary order are deduced, based on the lowest-order examples. These solutions can be useful for explanations of extremely large amplitude internal waves in the ocean, as well as for abnormally large-amplitude waves in other areas of nonlinear physics, such as optics and dusty plasmas.
\end{abstract}

DOI: 10.1103/PhysRevE.99.062224

\section{INTRODUCTION}

Internal waves are waves in layered structures. The latter include the atmosphere and the ocean, which are vital areas for human activity and for the existence of life itself. For example, layered structures in the world ocean are created due to stratifications in salinity, pressure, temperature, or underwater flows [1]. There can be sharp vertical density gradients, called pycnoclines. They can be due to flows (called "lee" or mountain waves) over topographical features on land.

Each type of stratification can be responsible for waves propagating along the corresponding interfaces. Knowledge of internal waves and their properties is important for the safe flight of airplanes in the atmosphere and the safe operation of submarines and other underwater devices in the ocean. In some cases, slow-moving ships are impeded because their engine energy is being transferred into an interfacial internal wave, although the water surface visibly stays calm. This phenomenon is known as the "dead water effect" [2], first noted in 1893.

Internal waves are well documented. In general, the layers of water have only a small density difference, and this means that the wave amplitudes tend to be higher than those for surface waves, while their velocities are a lot slower than those of surface waves [3]. They can be caused by tidal forces, the mixing of river fresh water with sea water [4], and from the energy put into water by the propellers or bow waves of ships. They have periods around 5-8 minutes and wavelengths in the range 0.6-1 km [5]. They can move oil production platforms [6].

Large-amplitude internal waves can be especially dangerous in this regard. They may not reveal themselves clearly on the ocean surface, but their vertical amplitudes underwater can exceed the amplitudes of rogue waves on the water surface. Indeed, their amplitudes can be as high as $170 \mathrm{~m}$ [7]. This value is more than 6 times the height of the "benchmark" surface rogue wave known as the "Draupner wave," as it

*mahyar.bokaeeyan@anu.edu.au was "only" $25.6 \mathrm{~m}$ high [8]. Internal waves of this amplitude may unexpectedly shift submarines down to a depth below the pressure capacity of the hull. They may have caused the Thresher submarine disaster in 1963 [6]. Possibly, the sinking was caused by a rogue internal wave with a high central amplitude [9]. Internal waves can also affect the transmission signals from submarines [10]. The existence of internal waves in the ocean has also been confirmed from space observations [11].

The term "rogue internal waves" was coined by Grimshaw et al. [12]. Several approaches have been developed in order to model rogue internal waves. They include the long-wave model described by the standard Korteweg-de Vries equation $(\mathrm{KdV})$ [12], a model based on a variable-coefficient $\mathrm{KdV}$ equation [13], and the one based on coupled nonlinear Schrödinger equations (cNLSE) [14], just to name a few.

In this paper, the mathematical model of internal waves is based on the Gardner equation (GE) considered in Ref. [12]. Numerical simulations made in Ref. [12] provided an indication that this equation may have solutions in the form of the rogue waves. Moreover, for the case of positive signs of all terms in this equation, its continuous-wave solution is subject to modulation instability, thus showing a similarity to the NLSE. The latter equation does have rogue-wave solutions represented by rational functions, as was shown in Ref. [15]. We can assume that rogue-wave solutions of GE would also be given by rational expressions. Here, we provide the first four rational solutions to the GE, showing localized wave amplification. As the GE is known to be applicable to threecomponent plasmas [16] and surface waves [17], the solutions that we find here can also be useful in these areas of physics.

The GE for the description of the internal waves can be written as:

$$
\psi_{x}+\alpha_{1} \psi \psi_{t}+\alpha_{2} \psi^{2} \psi_{t}+\delta \psi_{t t t}=0 .
$$

Here $\psi(x, t)$ is the normalized function of the amplitude of the wave, while subscripts $x$ and $t$ denote derivatives with respect to $x$ and $t$, respectively. The second and third terms are responsible for quadratic and cubic nonlinearities, respectively, 
and the last term denotes the third-order dispersion. Equation (1) sometimes is called a mixed $\mathrm{KdV}-\mathrm{mKdV}$ equation [18] or simply a modified $\mathrm{KdV}(\mathrm{mKdV})$ equation [12]. However, it is more common to call Eq. (4) below the $\mathrm{mKdV}$ equation. In order to avoid confusion, we use the most common name for Eq. (1), i.e., the "Gardner equation." This equation is more general than the $\mathrm{KdV}$ or $\mathrm{mKdV}$. When $\alpha_{1}=0$, the Gardner equation (1) reduces to the $\mathrm{mKdV}$ equation. When $\alpha_{2}=0$, it reduces to the $\mathrm{KdV}$ equation.

All the coefficients in (1) are defined by the density stratification and thickness of the layers. The coefficients $\alpha_{1}$ and $\delta$ are positive, while the sign of the coefficient $\alpha_{2}$ for internal waves depends on the stratification model $[14,19]$. In the case of a two-layer fluid, it is always negative [10]. However, in a three-layer model and in the case of more complicated stratification with shear flows, this coefficient can be positive $[20,21]$. In this paper, we restrict ourselves to this latter case. It has richer dynamics than the negative case [12]. Without loss of generality, the dependent and independent variables can be renormalized, thus setting each of the three coefficients to unity by a simple transformation [12]. In this way, Eq. (1) takes the following form:

$$
\psi_{x}+\psi \psi_{t}(1+\psi)+\psi_{t t t}=0
$$

An inverse rescaling transformation returns us to the original equation (1), and its solutions, with all three coefficients included. It is easy to show that if $\psi(x, t)$ is a solution of the Gardner equation, (2), then the function

$$
\hat{\psi}(x, t)=-\psi(x, t)-1
$$

is also a solution of the same equation. The importance of the transformation (3) should not be underestimated, as it relates bright and dark rogue waves of the GE. Below, we present both bright and dark rogue-wave solutions of Eq. (2).

\section{FIRST-ORDER RATIONAL SOLUTIONS OF THE GARDNER EQUATION}

The lowest-order exact rational solution to the GE can be obtained by using the Lorentz transformation [17] applied to the first $\mathrm{mKdV}$ rational solution found in Ref. [22]. (See Eq. (3) of Ref. [22] for $\beta=1$ and $\gamma_{3}=-1$.) Namely, let $u(x, t)$ be an exact solution to the $\mathrm{mKdV}$ equation,

$$
u_{x}+u^{2} u_{t}+u_{t t t}=0
$$

Then

$$
u^{\prime}(x, t)=k u\left(k^{3} x, k t\right)
$$

is also a solution, with $k$ being a real constant. This rescaling extends an individual solution to a one-parameter family of solutions with $k$ being the parameter of the family. Now, if we know any solution $u^{\prime}(x, t)$ of Eq. (4), the function $\psi(x, t)$ defined as

$$
\psi(x, t)=u^{\prime}\left(x, t+\frac{1}{4} x\right)-\frac{1}{2}
$$

is an exact solution of the GE, Eq. (2).

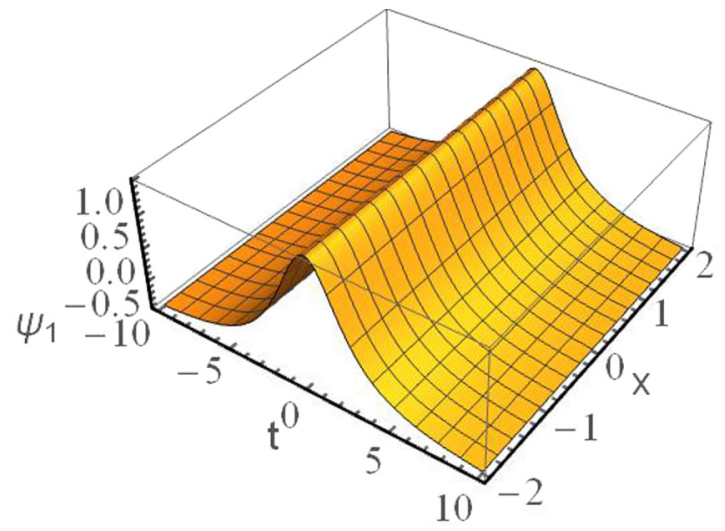

FIG. 1. The first-order bright rational solution of the Gardner equation is given by Eq. (8). Here the background is -1 . The maximum, at $t=0$, is 1 , thus giving an amplitude of 2 .

Namely, using the exact lowest-order rational solution of $\mathrm{mKdV}$ [22],

$$
u_{1}(x, t)=\frac{12}{3+2(t-x)^{2}}-1,
$$

and taking $k=\frac{1}{2}$ in (5), we obtain the exact lowest- (first-) order rational solution to the $\mathrm{GE}$,

$$
\psi_{1}(x, t)=\frac{12}{t^{2}+6}-1
$$

The form of this solution is shown in Fig. 1. The solution is stationary in $x$. The maximum amplitude relative to the background at $t=0$ is 2 while the background level at $t \rightarrow$ $\pm \infty$ is -1 . It may look like a zero velocity soliton with positive amplitude but we have to keep in mind that this is rational solution rather than the sech function. The solution (8) has been derived earlier by Grimshaw et al. in Ref. [13] as a limiting case of a sech-shaped soliton solution.

Now, using the transformation (3), we can easily find the inverted first-order solution:

$$
\hat{\psi}_{1}(x, t)=-\psi_{1}(x, t)-1=-\frac{12}{t^{2}+6},
$$

This solution is plotted in Fig. 2. This solution is also stationary in $x$. In contrast to the solution in Fig. 1, this one resembles a dark soliton. For this solution, the background level is 0 and the minimum is -2 .

Thus, we have two rational first-order solutions of the GE. One is given by Eq. (8) and the other by Eq. (9). This is not surprising because the transformation of Eq. (3) can be used with any solution of the GE, including rogue-wave solutions of higher order. This transformation also means that there are two types of rogue waves: "bright" ones, denoted $\psi_{j}(x, t)$, and "dark" ones, denoted $\hat{\psi}_{j}(x, t)$. Although their background levels differ, their amplitudes relative to the background level are the same.

To connect this with soliton ideas, we can write down the one-parameter family of soliton solutions of the GE for real parameter $\kappa$ :

$$
\psi_{s}(x, t)=\frac{1-\kappa^{2}}{\kappa\left\{\cosh \left[\frac{\sqrt{1-\kappa^{2}}\left(\kappa^{2}(6 t+x)-x\right)}{6 \sqrt{6} \kappa^{3}}\right]+\kappa\right\}} .
$$




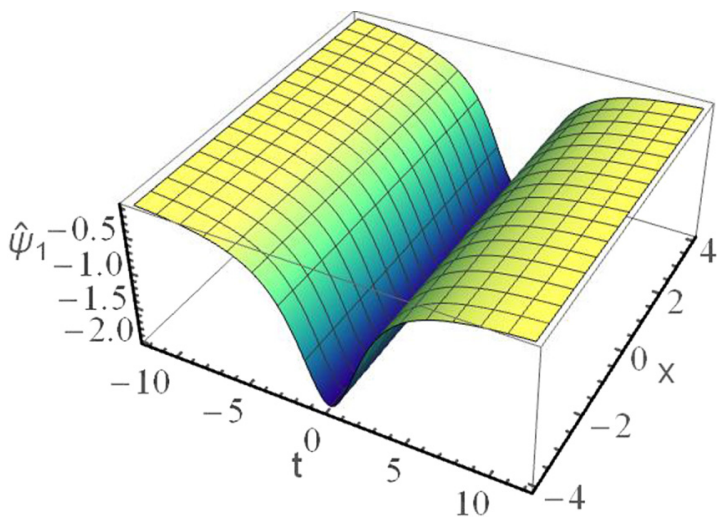

FIG. 2. The first-order dark rational solution of the Gardner equation from Eq. (9). Here the background level is 0. Minimum at $t=0$ is -2 . In order to isolate clearly dark rogue-wave solutions from bright ones, a distinct color scheme (yellow-green) is used for the dark rogue waves here and in all figures below.

In the limit $\kappa \rightarrow-1$, the solution (10) reduces to:

$$
\lim _{(\kappa \rightarrow-1)} \psi_{s}(x, t)=-\frac{12}{t^{2}+6},
$$

i.e., the first-order rational solution, Eq. (9).

\section{SECOND-ORDER RATIONAL SOLUTIONS AS ROGUE WAVES}

The general relation, Eq. (6), between the solutions of Gardner and $\mathrm{mKdV}$ equations allows us to construct higherorder rogue-wave solutions. The second-order rational solution of the $\mathrm{mKdV}$ equation has been obtained in Ref. [22] [see Eq. (5) therein]. Taking the coefficients of the $\mathrm{mKdV}$ equation in Ref. [22] to be $\beta=1$ and $\gamma_{3}=-1$ and using one of the above-mentioned transformations, we find that the second-order rational solution to the equation (2) is

$$
\psi_{2}(x, t)=-36 \frac{G_{2}}{D_{2}},
$$

where

$$
G_{2}=t^{4}+36 t^{2}-24 t x-108
$$

and

$$
\begin{aligned}
D_{2}= & 18\left(t^{4}-24 t x\right)+\left(t^{3}+12 x\right)^{2}+972 t^{2}+1944 \\
= & t^{6}+18 t^{4}+24\left(t^{2}-18\right) t x \\
& +972 t^{2}+144 x^{2}+1944,
\end{aligned}
$$

or, alternatively,

$$
\psi_{2}(x, t)=\frac{36\left(108-t^{4}-36 t^{2}+2 t X\right)}{\left(t^{3}-18 t+X\right)^{2}+54\left(t^{2}+6\right)^{2}},
$$

where $X=12 x$. This solution is shown in Fig. 3. It has a high peak that we can view as a rogue wave. Indeed, the peak is localized, both in time and in space, thus representing an unexpected extreme event. This event is clearly seen in Fig. 3. The background level of the solution, Eq. (13), is zero while the maximum amplitude of the rogue event is 2. The denominator of the solution in the form (13) is a

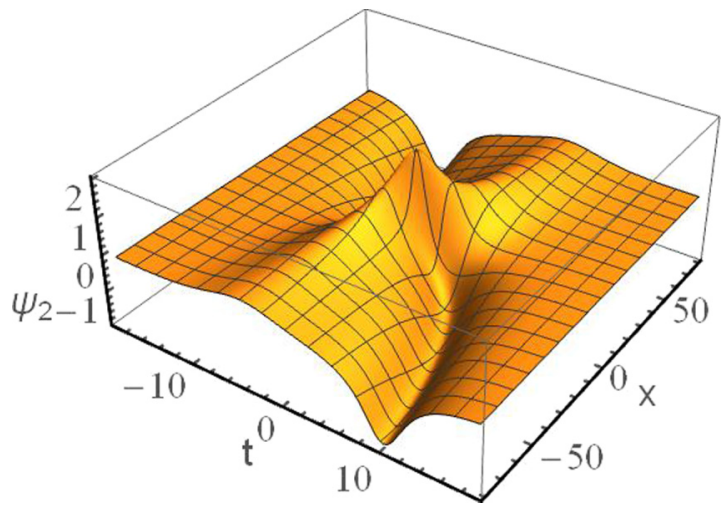

FIG. 3. The second-order rational solution of the GE given by Eq. (11). The "bright" rogue wave in the middle is clearly visible. Here the background is 0 . Maximum at the origin is 2 .

sum of squares. It is never zero, meaning that the solution is nonsingular. Such a form has been given in Refs. [23] and [24].

Using Eq. (3), we find the inverted second-order solution:

$$
\hat{\psi}_{2}(x, t)=36 \frac{G_{2}}{D_{2}}-1 .
$$

In contrast to the solution given by Eq. (13), the background level here is -1 and the extremal amplitude of this rogue wave is -3 . It is shown in Fig. 4. We call this solution a "dark rogue wave."

\section{THIRD-ORDER ROGUE WAVES}

Starting with the third-order solution of the $\mathrm{mKdV}$ given by Eq. (6) in Ref. [22] and using the Lorentz transformation, we are able to find the third rational solution of the GE:

$$
\psi_{3}(x, t)=72 \frac{G_{3}}{D_{3}}-1,
$$

where the numerator $G_{3}$ is

$$
\begin{aligned}
G_{3}= & t^{10}+90 t^{8}+7560 t^{6}-4320 t^{5} x \\
& +5400 t^{4}\left(x^{2}-18\right)+259200 t^{3} x \\
& -32400 t^{2}\left(2 x^{2}+27\right)+43200 t x\left(x^{2}+54\right) \\
& +194400\left(5 x^{2}+27\right),
\end{aligned}
$$

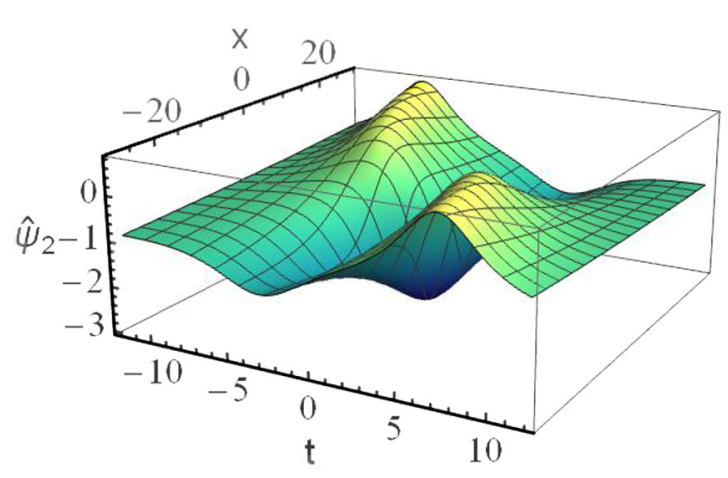

FIG. 4. The "dark" second-order rogue-wave solution given by Eq. (14). Here the background is -1 . The central minimum is -3 . 


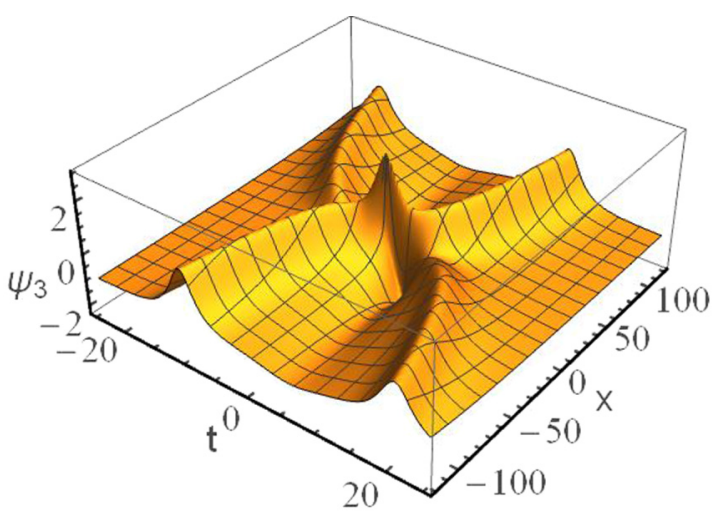

FIG. 5. Third-order rogue-wave solution of Gardner equation given by Eq. (15). Here the background level is -1 while the maximal amplitude at the origin is 3 .

while the denominator $D_{3}$ is

$$
\begin{aligned}
D_{3}= & t^{12}+36 t^{10}+120 t^{9} x+4860 t^{8} \\
& +2160 t^{6}\left(x^{2}+234\right)-233280 t^{5} x \\
& +97200 t^{4}\left(2 x^{2}+45\right)-86400 t^{3} x\left(x^{2}-108\right) \\
& -3499200 t^{2}\left(x^{2}-27\right)+777600 t x\left(2 x^{2}-135\right) \\
& +129600\left(4 x^{4}+594 x^{2}+729\right) .
\end{aligned}
$$

This solution is plotted in Fig. 5. It has the background level -1 and central amplitude 3 located at the origin due to the suitable choice of the coordinates.

Applying the transformation (3) to Eq. (15), we find the inverted third-order solution:

$$
\hat{\psi}_{3}(x, t)=-1-\psi_{3}(x, t)=-72 \frac{G_{3}}{D_{3}}
$$

with the same values of $G_{3}$ and $D_{3}$ as above. This solution is plotted in Fig. 6. The background level is now 0 and the minimum amplitude is -4 . The two local maxima appear to have amplitudes around $\psi_{\max } \approx 1.23$.

\section{THE FOURTH- AND HIGHER-ORDER ROGUE WAVES}

Applying the same technique as above to known higherorder rogue-wave solutions of $\mathrm{mKdV}$ equation, we can find

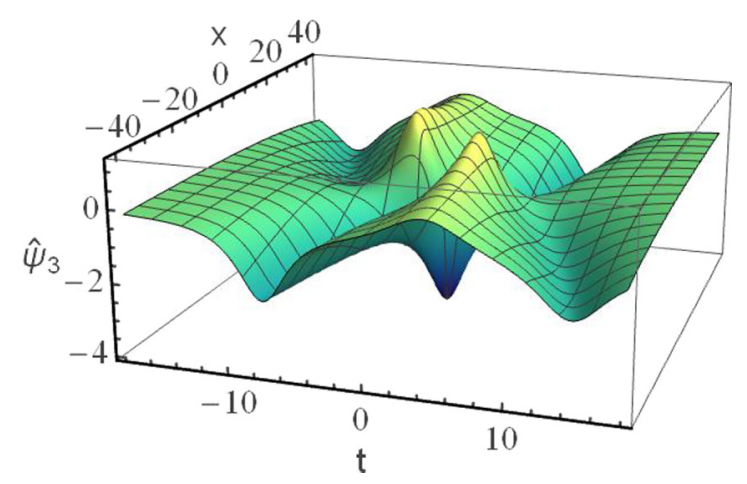

FIG. 6. "Dark" third-order rational solution to the GE given by Eq. (16). This solution is inverted relative to Fig. 5 and shifted down. Here the background is 0 . The central minimum is -4 .

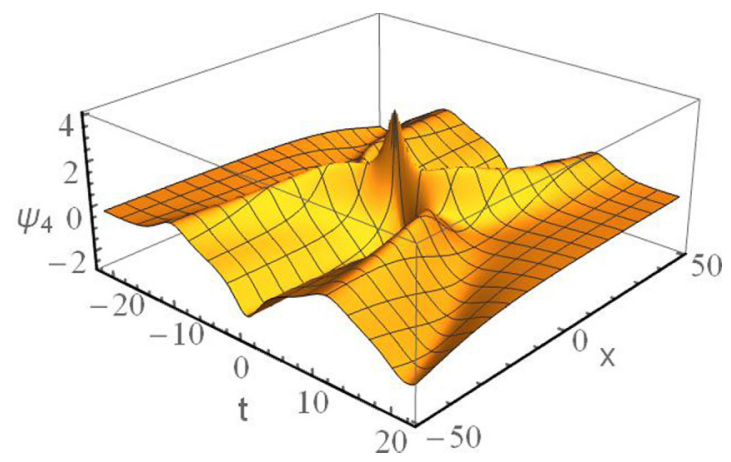

FIG. 7. Fourth-order "bright" rogue wave of the Gardner equation, given by Eq. (A1). Here the background level is 0. Maximal amplitude is 4 .

higher-order rogue-wave solutions of the Gardner equation. Explicit mathematical expressions of these solutions are quite involved and the degree of complexity increases with the order of the solution. Thus, we do not present the mathematical form of these solutions except for the fourth-order one which is given in the Appendix. In order to show that there are certain rules that these solutions obey, we only illustrate them using the next, fourth-order, solution. It is shown in Fig. 7. This solution also has a peak at the origin and its amplitude is 4 . The background level is 0. Finally, the inverted version of the fourth-order solution defined as

$$
\hat{\psi}_{4}(x, t)=-1-\psi_{4}(x, t) .
$$

is presented in Fig. 8. It has the background level -1 and the minimum at the origin is -5 .

\section{SUMMARY}

Having the first four rogue-wave solutions in explicit form, we are now in a position to predict the major characteristics of rogue waves of arbitrary order, $j$. Moreover, we are able to compare them with the features of rogue waves of the NLS and $\mathrm{mKdV}$ equations found in previous works, Refs. [25] and [22], respectively. In all three cases, the rogue-wave solutions are given in the form of rational solutions, and thus have much in common, even in this sense. Dimensionless forms

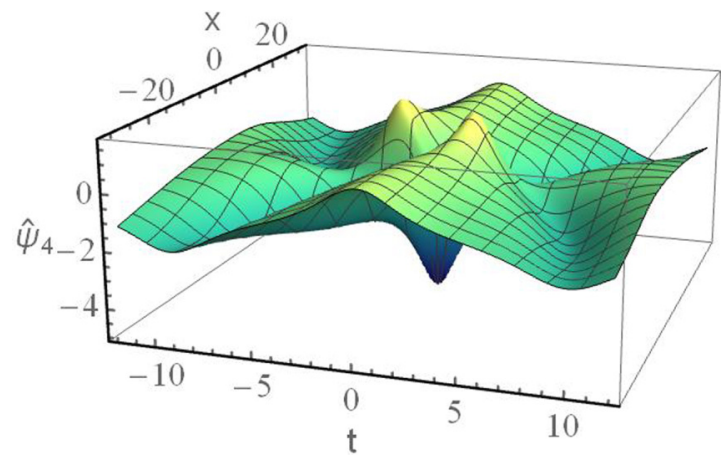

FIG. 8. "Dark" fourth-order rational solution of the GE, given by Eq. (17) and Eq. (A2). This solution is inverted relative to Fig. 7 and shifted down. Here the background is -1 . The central minimum is -5 . 
TABLE I. Main features (amplitude and the background level) of rogue waves of the NLSE [25], the mKdV [22], and the "bright rogue waves" $\left(\psi_{j}\right)$ of the Gardner equation. The $j$ th-order rogue wave of the Gardner equation has amplitude $j$, so, in the dimensionless form, the amplitude is much lower than the corresponding rogue waves of the mKdV equation and the NLSE.

\begin{tabular}{lcccc}
\hline \hline $\begin{array}{c}\text { The background level of the } \\
\text { NLS and mKdV rogue waves }\end{array}$ & $\begin{array}{c}\text { Maximal amplitude of the NLS } \\
\text { and mKdV rogue waves }\end{array}$ & $\begin{array}{c}\text { The background level of } \\
\text { the GE rogue waves }\end{array}$ & $\begin{array}{c}\text { Maximal amplitude of the } \\
\text { GE rogue waves }\end{array}$ \\
\hline 1 & -1 & 3 & -1 & 1 \\
2 & 1 & 5 & 0 & 3 \\
3 & -1 & 7 & 0 & 4 \\
4 & 1 & 9 & $\frac{1}{2}\left[(-1)^{j}-1\right]$ & $j$ \\
$j$ & $(-1)^{j}$ & $2 j+1$ & \\
\hline \hline
\end{tabular}

of these equations allow us to make purely mathematical comparisons. The real-world amplitudes are clearly another matter. The latter depend on particular parameters of the geometric structure of the layered media, and these may vary over a wide range.

One of the important predictions is related to the amplitude of rogue waves. Namely, the $j$ th member of the "bright" set of rogue waves, denoted $\psi_{j}(x, t)$, has a positive central maximum of $j$, while the $j$ th member of the "dark" set, denoted $\hat{\psi}_{j}(x, t)$, features a central minimum of amplitude $-j-1$.

The major difference between GE and $\mathrm{mKdV}$ rogue waves and those of the NLSE case is the presence of long tails, as can be seen from the plots above. They extend to infinity, thus modifying the background on which the rogue waves protrude with the higher amplitude. The presence of the tails may serve as a tool for predicting internal rogue waves much earlier than in the case of NLSE rogue waves, which lack such tails [15].

The presence of the central peak is the common feature in all three cases ( $\mathrm{mKdV}, \mathrm{GE}$, and NLSE), so a comparison of their amplitudes relative to the background levels (amplification factor) may provide further ideas for research on rogue waves. In Table I, we summarize these characteristics for hierarchies of rogue waves with increasing order, for the NLSE, taken from [25], the mKdV equation, taken from Ref. [22] and the bright rogue waves of the Gardner equation found in the present work. Further, in Table II, we summarize the characteristics of dark rogue waves of the Gardner equation, also found in the present work. In the "dark roguewave case," the highest magnitude amplitudes are naturally negative, as they represent waves of depression, while "bright rogue waves" show elevations.

Clearly, the next question to answer is the robustness of these solutions. We leave this problem aside here, as it

TABLE II. Main characteristics (background level and amplitude) of "dark rogue waves" $\left(\hat{\psi}_{j}\right)$ of the Gardner equation.

\begin{tabular}{lcc}
\hline \hline$j$ & $\begin{array}{c}\text { Background level of } \\
\text { "dark rogue waves" of GE }\end{array}$ & $\begin{array}{c}\text { Minimal amplitude of } \\
\text { "dark rogue waves" of GE }\end{array}$ \\
\hline 1 & 0 & -2 \\
2 & -1 & -3 \\
3 & 0 & -4 \\
4 & -1 & -5 \\
$j$ & $\frac{1}{2}\left[-1-(-1)^{j}\right]$ & $-(j+1)$ \\
\hline \hline
\end{tabular}

requires a complicated approach. Even the first-order solutions require detailed analysis before any conclusions can be drawn. The higher-order solutions are much more involved and may need more effort to understand their significance. All this cannot be done in a single work. Besides, in order to study robustness, the solutions have to be known in the first place. This first step is the fundamental one and it has been given in the present work. We should also note that all solutions, including unstable ones, play significant roles in the complex chaotic dynamics that may occur in layered systems. Thus, knowledge of the form of the whole set of exact solutions of the Gardner equation, whether stable or not, is crucial for further progress in this field.

Rogue-wave solutions are general features of many evolution equations and are not just "isolated" examples. They have been found for the NLSE [15], vector NLSE [26,27], Sasa-Satsuma equation [28], three-wave resonant interaction problem [29], Davey-Sewartson equations [30,31], and many other systems. These solutions are an essential part of the general waveform evolution in nonlinear systems, as the recent works [32,33] have shown. This addition of a new hierarchy of rogue waves for the Gardner equation will further expand our knowledge in this exciting field of research.

\section{ACKNOWLEDGMENTS}

The authors gratefully acknowledge the support of the Australian Research Council (Discovery Project DP150102057). M.B. is grateful to Dr. Wonkeun Chang for his technical support and helpful discussions.

\section{APPENDIX}

The two fourth-order rogue-wave solutions of the Gardner equation are given by:

$$
\psi_{4}(x, t)=-120 \frac{G_{4}}{D_{4}}
$$

for the bright rogue wave and

$$
\hat{\psi}_{4}(x, t)=120 \frac{G_{4}}{D_{4}}-1
$$

for the dark one. In each case,

$$
\begin{aligned}
G_{4}= & t^{18}+162 t^{16}+144 t^{15} x+32400 t^{14} \\
& +22680 t^{12}\left(x^{2}+180\right)-544320 t^{11} x \\
& +2449440 t^{10}\left(x^{2}-57\right)-2116800 t^{9} x\left(x^{2}-324\right)
\end{aligned}
$$




$$
\begin{aligned}
& -12247200 t^{8}\left(35 x^{2}+306\right) \\
& +97977600 t^{7} x\left(2 x^{2}+27\right) \\
& -76204800 t^{6}\left(2 x^{4}-81 x^{2}+729\right) \\
& -1371686400 t^{5} x\left(7 x^{2}-108\right) \\
& -138883248000 t^{4}\left(5 x^{2}-36\right) \\
& -123451776000 t^{3} x\left(2 x^{2}+81\right) \\
& +10287648000 t^{2}\left(8 x^{4}+378 x^{2}+3645\right) \\
& -1111065984000 t x\left(5 x^{2}+108\right) \\
& +2286144000\left(4 x^{6}+1296 x^{4}+2187 x^{2}-19683\right)
\end{aligned}
$$

and

$$
D_{4}=t^{20}+60 t^{18}+360 t^{17} x+14580 t^{16}+8640 t^{15} x
$$

$$
\begin{aligned}
& +32400 t^{14}\left(x^{2}+108\right)+1360800 t^{12}\left(x^{2}+405\right) \\
& +604800 t^{11} x\left(x^{2}-162\right)+220449600 t^{10}\left(x^{2}+58\right) \\
& -127008000 t^{9} x\left(x^{2}-405\right) \\
& +27216000 t^{8}\left(4 x^{4}-1215 x^{2}+29889\right) \\
& +17635968000 t^{7} x\left(x^{2}-27\right) \\
& -1143072000 t^{6}\left(8 x^{4}-1215 x^{2}-32076\right) \\
& -740710656000 t^{5} x\left(x^{2}+54\right) \\
& +4166497440000 t^{4}\left(14 x^{2}+45\right) \\
& -11110659840000 t^{3} x\left(5 x^{2}-54\right) \\
& +22861440000 t^{2}\left(4 x^{6}+648 x^{4}-24057 x^{2}+98415\right) \\
& +33331979520000 t x\left(14 x^{2}-135\right) \\
& +68584320000\left(8 x^{6}+3240 x^{4}+83106 x^{2}+19683\right) .
\end{aligned}
$$

[1] C. Garrett, Internal tides and ocean mixing, Science 301, 1858 (2003).

[2] T. Miloh, M. P. Tulin and G. Zilman, Dead-water effects of a ship moving in stratified seas, J. Offshore Mech. Arct. Eng. 115, 105 (1993).

[3] M. Grant Gross, Oceanography (Prentice-Hall, Piscataway, NJ, 1972).

[4] J. Weisberg and H. Parish, Introductory Oceanography (McGraw-Hill, New York, 1974).

[5] H. Thurman and E. Burton, Introductory Oceanography, 10th ed. (Prentice-Hall, Piscataway, NJ, 2004).

[6] D. A. Segar, Introduction to Ocean Sciences, 2nd ed. (W. W. Norton \& Co., NY, 2007).

[7] M. H. Alford, The formation and fate of internal waves in the South China Sea, Nature 521, 65 (2015).

[8] L. Cavaleri, F. Barbariol, A. Benetazzo, L. Bertotti, J.-R. Bidlot, P. Janssen, N. Wedi, The Draupner wave: A fresh look and the emerging view, J. Geophys. Res.: Oceans 121, 6061 (2016).

[9] J. Grue, Very large internal waves in the ocean-Observations and nonlinear models, in: Waves in Geophysical Fluids, Tsunamis, Rogue Waves, Internal Waves and Internal Tides, edited by J. Grue and K. Trulsen, Springer Courses and Lectures (Springer, NY, 2006), Vol. 489, pp. 205-270.

[10] J. R. Apel, L. A. Ostrovsky, Y. A. Stepanyants, and J. F. Lynch, Internal solitons in the ocean and their effect on underwater sound, J. Acoust. Soc. Am. 121, 695 (2007).

[11] Q. Zheng, V. Klemas, and X.-H. Yan, Advance in studies of ocean internal waves observed from space Res. Signpost 4, 143 (2002).

[12] R. Grimshaw, E. Pelinovsky, T. Taipova, and A. Sergeeva, Rogue internal waves in the ocean: Long wave model, Eur. Phys. J. Spec. Top. 185, 195 (2010).

[13] R. Grimshaw, E. Pelinovsky, and T. Talipova, The modified Korteweg-de Vries equation in the theory of large-amplitude internal waves, Nonlin. Process. Geophys. 4, 237 (1997).

[14] R. Grimshaw, E. Pelinovsky, and T. Talipova, Modelling internal solitary waves in the coastal ocean, Surv. Geophys. 28, 273 (2007).
[15] N. Akhmediev, A. Ankiewicz, and M. Taki, Waves that appear from nowhere and disappear without a trace, Phys. Lett. A 373, 675 (2009).

[16] M. A. Rehman, and M. K. Mishra, Ion-acoustic Gardner Solitons in electron-positron-ion plasma with two-electron temperature distributions, Phys. Plasmas 23, 012302 (2016).

[17] A. M. Kamchatnov, Y.-H. Kuo, T.-C. Lin, T.-L. Horng, S.-C. Gou, R. Clift, G. A. El, and R. H. J. Grimshaw, Undular bore theory for the Gardner equation, Phys. Rev. E 86, 036605 (2012).

[18] T. Kakutani and N. Yamasaki, Solitary waves on a two layer fluid, J. Phys. Soc. Jpn. 45, 674 (1978).

[19] M. Li, J.-H. Xiao, M. Wang, Y.-F. Wang, and Bo Tian, Solitons for a forced extended Korteweg-de Vries equation with variable coefficients in atmospheric dynamics, Z. Naturforsch. A 68, 235 (2013).

[20] R. Grimshaw, E. Pelinovsky, Y. Stepanyants, T. Talipova, Modeling internal solitary waves on the Australian North West Shelf, Mar. Freshw. Res. 57, 265 (2006).

[21] L. A. Ostrovsky, E. N. Pelinovsky, V. I. Shrira and Y. A. Stepanyants, Beyond the KDV: Post-explosion development, Chaos 25, 097620 (2015).

[22] A. Ankiewicz, and N. Akhmediev, Rogue wave-type solutions of the $\mathrm{mKdV}$ equation and their relation to known NLSE rogue wave solutions, Nonlin. Dynam. 91, 1931 (2018).

[23] D. E. Pelinovsky, R. H. J. Grimshaw, Structural transformation of eigenvalues for a perturbed algebraic soliton potential, Phys. Lett. A 229, 165 (1997).

[24] M. J. Ablowitz, and J. Satsuma, Solitons and rational solutions of nonlinear evolution equations, J. Math. Phys. 19, 2180 (1978).

[25] N. Akhmediev, A. Ankiewicz, and J. M. Soto-Crespo, Rogue waves and rational solutions of the nonlinear Schrödinger equation, Phys. Rev. E 80, 026601 (2009).

[26] F. Baronio, A. Degasperis, M. Conforti, and S. Wabnitz, Solutions of the Vector Nonlinear Schrödinger Equations: Evidence for Deterministic Rogue Waves, Phys. Rev. Lett. 109, 044102 (2012). 
[27] N. Vishnu Priya, M. Senthilvelan and M. Lakshmanan, Akhmediev breathers, Ma solitons, and general breathers from rogue waves: A case study in the Manakov system, Phys. Rev. E 88, 022918 (2013).

[28] U. Bandelow and N. Akhmediev, Persistence of rogue waves in extended nonlinear Schrödinger equations: Integrable SasaSatsuma case, Phys. Lett. A 376, 1558 (2012).

[29] F. Baronio, A. Degasperis, M. Conforti, and S. Lombardo, Rogue Waves Emerging from the Resonant Interaction of Three Waves, Phys. Rev. Lett. 111, 114101 (2013).

[30] Y. Ohta and J. K. Yang, Rogue waves in the Davey-Stewartson I equation, Phys. Rev. E 86, 036604 (2012).
[31] Y. Ohta and J. K. Yang, Dynamics of rogue waves in the DaveyStewartson II equation, J. Phys. A: Math. Theor. 46, 105202 (2013).

[32] A. Tikan, C. Billet, G. El, A. Tovbis, M. Bertola, T. Sylvestre, F. Gustave, S. Randoux, G. Genty, P. Suret, and J. M. Dudley, Universality of the Peregrine Soliton in the Focusing Dynamics of the Cubic Nonlinear Schrödinger Equation, Phys. Rev. Lett. 119, 033901 (2017).

[33] B. Kibler, J. Fatome, C. Finot, G. Millot, F. Dias, G. Genty, N. Akhmediev, and J. M. Dudley, The Peregrine soliton in nonlinear fibre optics, Nat. Phys. 6, 790 (2010). 